\title{
Performance Analysis of Multihop Relaying Caching for Internet of Things under Nakagami Channels
}

\author{
Baofeng Ji, ${ }^{1,2}$ Bingbing Xing, ${ }^{1}$ Kang Song, ${ }^{3}$ Chunguo Li $\mathbb{D}^{1,4}$ Hong Wen, ${ }^{2}$ and Luxi Yang ${ }^{4}$ \\ ${ }^{1}$ School of Information Engineering, Henan University of Science and Technology, Luoyang 471023, China \\ ${ }^{2}$ University of Electronic Science and Technology of China, Chengdu 611731, China \\ ${ }^{3}$ School of Information Engineering, Qingdao University, Qingdao 266000, China \\ ${ }^{4}$ School of Information Science and Engineering, Southeast University, Nanjing 210096, China
}

Correspondence should be addressed to Chunguo Li; chunguoli@seu.edu.cn

Received 28 December 2017; Revised 7 February 2018; Accepted 27 February 2018; Published 23 April 2018

Academic Editor: Nan Zhao

Copyright (c) 2018 Baofeng Ji et al. This is an open access article distributed under the Creative Commons Attribution License, which permits unrestricted use, distribution, and reproduction in any medium, provided the original work is properly cited.

\begin{abstract}
Performance analysis is studied in this paper for the wireless transmissions in Internet of Things (IoT) system, where both the direct link and the multihop relaying caching wireless transmission from the source node to the destination node are taken into the consideration. The key feature is the Nakagami channels of the wireless channel from the source node to the destination node, which results in the difficulty of the theoretical analysis over the system performance. To tackle this difficulty, the probability distribution function (PDF) of the received signal-to-noise ratio (SNR) at the destination node is derived by exploiting the function and integral properties. Then, the outage probability and bit error rate (BER) of the whole wireless IoT system are derived in the analytical expression without any approximation. Numerical simulations demonstrate the accuracy of the derived theoretical analysis for this system.
\end{abstract}

\section{Introduction}

Internet of Things (IoT) becomes very import since it can support the requirement from the fifth generation (5G) of the cellular transmission. For instance, IoT can serve massive access nodes for wireless service in the scenario of the ultradense networks (UDN) in 5G [1-4]. It is shown that there are as many as over three thousands of papers in IEEE exploring over the study of IoT, where the number of the journal papers is over six hundreds. For example, RFID as IoT enabler is reviewed in [5] from both the physical layer and MAC layer point of review, where the SDR (soft defined radio) platform is utilized to guarantee the time delay of the tag identification. In [6], the performance of employee is evaluated automatically by the proposed game theories, where the data is collected by the sensory nodes in IoT systems. Three deployment patterns of the industrial IoT are theoretically discussed by the authors from [7] for the cognitive access in IoT. The cost and the performance tradeoff are demonstrated in [8-10] for selecting the platform in IoT, where the CUP, GPU, and FPGA are taken into the consideration for the computer vision. In [11], the smart manufacturing with real-time traceability is studied in [11] which is enabled by IoT, where the authors propose a production performance analysis and exception diagnosis model. The authors in [12] review the channel access protocols for IoT system, where the ALOHA method is discussed in detail. All of these research results show that IoT have potential good performance for wireless performance especially in $5 \mathrm{G}$ cellular systems. However, there is no study on the outage probability analysis over IoT where the wireless channel from the source node to the destination node is distributed with the Nakagami.

There are extensive results over the performance analysis in existing wireless communication systems especially in the multihop relaying system without involving IoT technology. The multihop system with cooperative relay is usually named as multihop cooperative relay systems (MCRSs). Compared to traditional networks, MCRSs have a lot of advantages such as the connectivity improvement and the capacity enhancement. In multihop wireless communications, many relay stations assist the source terminal to transmit signal to 
the destination terminal, which reduces the transmit power and improves the wireless link reliability especially for the scenario of the long-distance between the source and the destination [6-13]. The amplify-and-forward relaying protocol is studied in [13] for the multihop and multibranch wireless cooperative systems with nonregenerative fixed gain at the relay station. In [14], the outage probability and the bit error rate are analyzed theoretically for MCRSs over Nakagami$m$ fading channels, where the approximated expression is derived in closed form. The asymptotic performance is analyzed in [15] for the bit error rate in the multihop relaying caching networks, where each hop of the wireless channel is distributed with Generalized-K. These studies are applicable to the amplify-and-forward (AF) relaying protocol. A method is proposed in [16] which is based on the generalized transformed characteristic function (GTCF) for the AF multihop relaying system. A statistical-theorem-based approach is proposed to analyze the performance of multihop relaying system in [17]. For the decode-and-relaying protocol, the ergodic capacity is theoretically analyzed in [18-20]. It is discovered from [19] that the fixed AF relaying method outperforms the other relaying methods such as the fixed DF and the selective DF. All these studies are suitable for one-way relaying protocol, where the two-way relaying protocol is examined in [20] by deriving the expression to the outage probability. The dual-hop wireless communication system has been theoretically analyzed in [21-23], where performance analysis is derived for different assumptions. However, all of these results cannot be applied to the more general scenario where the wireless channel is distributed with the Nakagami, where the goal is to obtain the accurate expression without approximation for the target performance such as the outage probability and the bit error rate.

With the fifth generation mobile communication $(5 \mathrm{G})$, the Internet of Things (IoT) technology becomes more and more important since IoT can provide many attractive improvements to 5G. The IoT-based wireless communications typically employ the relaying technique which can enlarge the coverage of the wireless communications. For instance, the mobile terminal in the vehicle-based relaying system transmits signals to the base station via the multihop relaying retransmission [24]. A dual interface relay is designed to improve the power efficiency of the IoT-based wireless sensor networks; it is proved in [25] that the relaying technology reduces the outage probability in the energy harvesting IoT-based wireless networks while decreasing the feedback cost from the receive node to the transmit node. Thus, it is interesting to study the performance analysis over the relaying based IoT wireless transmissions.

In this paper, the signal-to-noise ratio (SNR) from the source to the destination is derived in the closed form for the IoT wireless communications. Moreover, the outage probability and the bit error rate are theoretically analyzed by deriving the analytical expression via exploiting the probability properties. The key feature is that all wireless links are distributed with Nakagami- $m$ from the source node to the destination node. The final expression over the outage probability is exact without any approximation for the studied IoT wireless system.

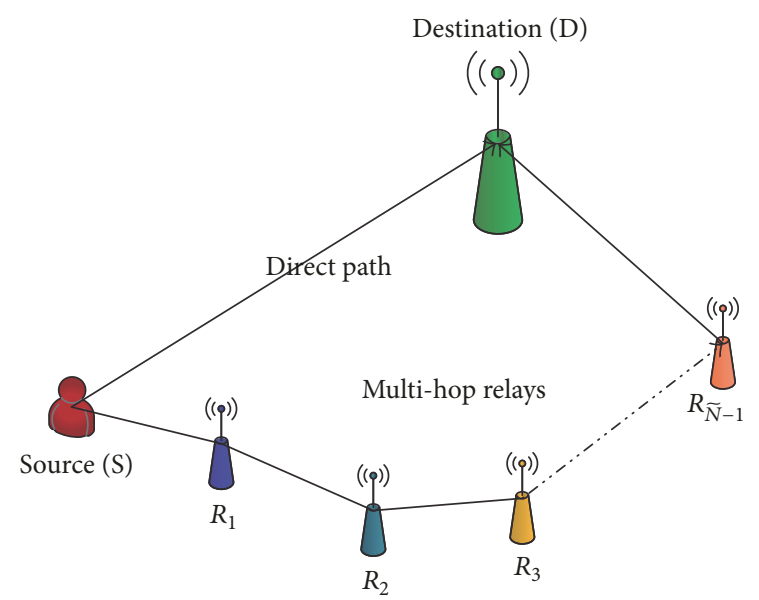

FIGURE 1: Illustration of a multihop cooperative relay network.

The rest of this paper is organized as follows. The system model is introduced in Section 2 for the IoT multihop relaying wireless communications with the existence of the direct wireless link from the source to the destination node. In Section 3, the probability density function (PDF) of multihop relaying link is derived exactly in an analytical expression. Moreover, the outage probability and the bit error rate are derived by exploiting the probability properties, which are based on the derived PDF as mentioned above. In Section 4, the numerical simulations are plotted to show the effectiveness of the obtained theoretical analysis. Section 5 concludes the whole paper.

\section{System Model}

The considered system consisting of one source node, one destination, and $\bar{N}-1$ relay nodes as shown in Figure 1 . In the first time slot, the source node transmits the signal to the first relay node. Meanwhile, the destination also receives the signal from the source node by the wireless channel, which is usually ignored by most of the existing methods in this literature. In the $\bar{n}$ th $(\bar{n}=2,3, \ldots, \bar{N}-1)$ time slot, the $\bar{n}-1$ th relay node first amplifies the received caching signal and the retransmits it to the $\bar{n}$ th relay node. Finally, the $\bar{N}$ th relay node amplifies the received caching signal and forwards it to the destination in the $\bar{N}$ th time slot. At the destination, the receiver receives two branches of the signals, respectively, from the source node directly and from the relay nodes via multihop caching technology. The maximal-ratio combining (MRC) is employed at the destination node to combine these two branches, which maximizes the signal-to-noise ratio at the destination. It is assumed that the channel is slow fading or block fading in the multihop IoT networks.

In the first time slot, the source node as the transmitter in IoT wireless networks sends the signal via the wireless channel to the destination node directly. The received signal $y_{D}$ at the destination node $D$ is then given by

$$
y_{D}=\sqrt{P_{S}} \mathbf{h} s+n
$$


where $P_{S}$ is the transmission power of the source terminal, h denotes the channel matrix from transmitting antenna of source terminal to receiving antenna of the destination terminal, and the channel $h$ follows Nakagami- $m$ distribution and the norm of channel $h$, which means that the signal with shadowing effect can be approximated by a gamma distribution as $f_{\|h\|^{2}}(x)=(1 / \Gamma(\lambda))(\lambda / \Omega)^{\lambda} x^{\lambda-1} e^{-(\lambda / \Omega) x}, x>0 . s$ is the source transmitted signal, $n$ is additive white Gaussian noise (AWGN) at the destination, and $n \sim C N\left(0, \sigma_{n}^{2}\right)$.

In the following time slots, the relay node amplifies the received caching signal and then forwards it to the next relay node. The received caching signal $y_{1}$ at the 1 th relay terminal can be described as

$$
y_{1}=\sqrt{P_{S}} \mathbf{h}_{1} s+n_{1},
$$

where $\mathbf{h}_{1}$ denotes the channel matrix from transmitting antenna of source terminal to the receiving antenna of 1th relay terminal, $s$ is the source transmitted signal, $n_{1}$ is additive white Gaussian noise (AWGN) at the 1th relay terminal, and $n_{1} \sim C N\left(0, \sigma_{N}^{2}\right)$. In order to satisfy the requirements of relay transmission power, the signal receiving energy is normalized at the relay (divided by $\rho=\sqrt{P_{S}\left\|\mathbf{h}_{1}\right\|_{2}^{2}+\sigma_{n}^{2}}$ ) and amplified at the current relay node with the factor $w_{r},\left|w_{r}\right|=\sqrt{P_{r}}$. It is noting that the channel parameter follows Nakagami distribution, where every signal is subject to independent path loss, shadowing, and Nakagami fading effect. The reasons to choose the Nakagami- $m$ channels in the paper are as follows.

(1) Nakagami- $m$ channel characteristic can conform practical experience data more exactly than Rayleigh and Rice channel; it is worth noting that Nakagami- $m$ channel can accurately feature terrestrial wireless communication channel, indoor mobile communication channel, and other wireless multipath fading channels [26].

(2) Nakagami- $m$ channel can be equivalent to other wireless multipath fading channels with different parameter $m \in(1 / 2, \infty)$, which present that Nakagami- $m$ channel can cover other fading channels generally. In other words, Nakagami channel model has become a general channel model and thus has a high application value.

Nakagami- $m$ channel

$$
\Longrightarrow \begin{cases}\text { Unilateral Gaussian channel, } & m=\frac{1}{2} \\ \text { Rayleigh channel, } & m=1 \\ \text { Nakagami- } n, m=\frac{\left(1+n^{2}\right)^{2}}{1+2 n^{2}}, & n \geq 0 \\ \text { Ricechannel, } K=n^{2}=\frac{\sqrt{m^{2}-m}}{m-\sqrt{m^{2}-m}}, & m \geq 1 \\ \text { Constant channel, } & m=\infty .\end{cases}
$$

Thus, the transmitting caching signal of 1th relay node is expressed as

$$
t=\frac{w_{r}}{\rho} y_{1}
$$

Similarly, the receiving signal of 2 th relay node is given by

$$
\begin{aligned}
y_{2} & =\mathbf{h}_{2} \cdot t+n_{2}=\mathbf{h}_{2}\left(\frac{w_{r}}{\rho} y_{1}\right)+n_{2} \\
& =\mathbf{h}_{2} \frac{w_{r}}{\rho} \sqrt{P_{s}} \mathbf{h}_{1} s+\mathbf{h}_{2} \frac{w_{r}}{\rho} n_{1}+n_{2},
\end{aligned}
$$

where $\mathbf{h}_{2}$ is the channel matrix from the transmit antenna of 1 th relay node to the receive antenna of 2 th relay node and $n_{2}$ is additive white Gaussian noise (AWGN) at the 2th relay node and $n_{2} \sim C N\left(0, \sigma_{n}^{2}\right)$. In this way, the signal received at the $\bar{n}+1$ th $(\bar{n} \leq \bar{N})$ relay node is expressed as

$$
\begin{aligned}
y_{\bar{n}}= & \mathbf{h}_{\bar{n}}\left(\frac{w_{r}}{\rho} y_{\bar{n}-1}\right)+n_{\bar{n}} \\
= & \left(\frac{w_{r}}{\rho}\right)^{\bar{n}-1} \mathbf{h}_{\bar{n}} \mathbf{h}_{\bar{n}-1} \cdots \mathbf{h}_{1} \sqrt{P_{s}} s \\
& +\left(\frac{w_{r}}{\rho}\right)^{\bar{n}-1} \mathbf{h}_{\bar{n}} \mathbf{h}_{\bar{n}-1} \cdots \mathbf{h}_{2} n_{1} \\
& +\left(\frac{w_{r}}{\rho}\right)^{\bar{n}-2} \mathbf{h}_{\bar{n}} \mathbf{h}_{\bar{n}-1} \cdots \mathbf{h}_{3} n_{2}+\cdots+\frac{w_{r}}{\rho} \mathbf{h}_{\bar{n}} n_{\bar{n}-1} \\
& +n_{\bar{n}},
\end{aligned}
$$

where $\mathbf{h}_{\bar{n}}, \mathbf{h}_{\bar{n}-1}, \ldots, \mathbf{h}_{1}$ are the wireless channel vectors, $n_{\bar{n}}, n_{\bar{n}-1}, \ldots, n_{1}$ is the AWGN noise received at the corresponding relay node, and $n_{\bar{n}} \sim \operatorname{CN}\left(0, \sigma_{n}^{2}\right), \ldots, n_{1} \sim$ $C N\left(0, \sigma_{n}^{2}\right)$.

\section{Performance Analysis for IoT-Based Multihop Caching}

In this section, the signal-to-noise ratio is analyzed theoretically by deriving the corresponding probability density function (PDF), where the goal is to obtain the closed form expression. Then, the outage probability and the bit error rate are derived by utilizing the integration properties.

For the destination, there are two branches of the received signals that are directly from the source node and from the multihop caching node. With the signal arrived at the destination node from the multihop relaying caching link [27], the PDF of the corresponding SNR over the multihop caching link is given by

$$
\begin{aligned}
& f_{\text {Multhop }}(y) \\
& \quad=\frac{\partial\left\{1-\prod_{\bar{n}=1}^{\bar{N}}\left(1-\Gamma\left(m_{\bar{n}}, m_{\bar{n}} y / \bar{\gamma}_{\bar{n}}\right) / \Gamma\left(m_{\bar{n}}\right)\right)\right\}}{\partial y},
\end{aligned}
$$

where symbol $\partial(\cdot)$ is the derivation to variables and the function $\Gamma(\cdot, \cdot)$ is Gamma function and defined as $\Gamma(z)=$ $\int_{0}^{\infty} e^{-t} t^{z-1} d t[28]$, which can be sorted as

$$
\Gamma\left(m_{\bar{n}}, \frac{m_{\bar{n}} y}{\bar{\gamma}_{\bar{n}}}\right)=\Gamma\left(m_{\bar{n}}\right) \cdot e^{-m_{\bar{n}} y / \bar{\gamma}_{\bar{n}}} \cdot \sum_{j=0}^{m_{\bar{n}}-1} j !\left(\frac{m_{\bar{n}} y}{\bar{\gamma}_{\bar{n}}}\right)^{j} .
$$


It can be mentioned that the parameter $m$ needs to calculate in the form of integer in order to derive conveniently. By inserting (8), we have that

$$
\begin{aligned}
& f_{\text {Multihop }}(y) \\
& \quad=-\frac{\partial\left\{\prod_{\bar{n}=1}^{\bar{N}}\left(1-e^{-m_{\bar{n}} y / \bar{\gamma}_{\bar{n}}} \cdot \sum_{j=0}^{m_{\bar{n}}-1} j !\left(m_{\bar{n}} y / \bar{\gamma}_{\bar{n}}\right)^{j}\right)\right\}}{\partial y} .
\end{aligned}
$$

It is seen from (9) that the multiplication is involved in many terms, which makes the following derivations intractable. Thus, we propose to express (9) into another form equivalently, where the term involving the multiplication operation in (9) is derived as

$$
\begin{aligned}
& \prod_{\bar{n}=1}^{\bar{N}}\left(1-e^{-m_{\bar{n}} y / \bar{\gamma}_{\bar{n}}} \cdot \sum_{j=0}^{m_{\bar{n}}-1} j !\left(\frac{m_{\bar{n}} y}{\bar{\gamma}_{\bar{n}}}\right)^{j}\right) \\
& \quad=\sum_{t=0}^{\bar{N}} \sum_{b_{1} b_{2} \cdots b_{t} \in\left\{a_{1} a_{2} \cdots a_{\bar{N}}\right\} ; \forall k, l \in\{1,2, \ldots, t\}, b_{k} \neq b_{l}}(-1)^{t} b_{1} b_{2} \cdots b_{t} .
\end{aligned}
$$

$f_{\text {Multihop }}(y)$

$$
\begin{aligned}
& =\frac{\partial\left\{\sum_{t=0}^{\bar{N}} \sum_{\mho} \sum_{j_{1}=0}^{m_{1}-1} \sum_{j_{2}=0}^{m_{2}-1} \cdots \sum_{j_{t}=0}^{m_{t}-1}\left(\left(\left(m_{1} / \bar{\gamma}_{1}\right)^{j_{1}} \cdots\left(m_{t} / \bar{\gamma}_{t}\right)^{j_{t}} \cdot y^{\sum_{q=1}^{t} j_{q}}\right) /\left((-1)^{t+1} \cdot e^{y \cdot \sum_{p=1}^{t}\left(m_{p} / \bar{\gamma}_{p}\right)} \cdot j_{1} ! \cdots j_{t} !\right)\right)\right\}}{\partial y} \\
& =\sum_{t=0} \sum_{\mho} \sum_{j_{1}=0}^{m_{1}-1} \sum_{j_{2}=0}^{m_{2}-1} \cdots \sum_{j_{t}=0}^{m_{t}-1} \frac{\left(m_{1} / \bar{\gamma}_{1}\right)^{j_{1}} \cdots\left(m_{t} / \bar{\gamma}_{t}\right)^{j_{t}}}{(-1)^{t+1} \cdot j_{1} ! \cdots j_{t} !} \\
& \cdot\left\{y^{-1+\sum_{q=1}^{t} j_{q}} \cdot e^{-y \cdot \sum_{p=1}^{t}\left(m_{p} / \bar{\gamma}_{p}\right)} \cdot \sum_{q=1}^{t} j_{q}-\sum_{p=1}^{t} \frac{m_{p}}{\bar{\gamma}_{p}} \cdot y^{\sum_{q=1}^{t} j_{q}} \cdot e^{-y \cdot \sum_{p=1}^{t} m_{p} / \bar{\gamma}_{p}}\right\} .
\end{aligned}
$$

In order to expressed clearly and simply, it can be denoted that $b_{1} b_{2} \cdots b_{t} \in\left\{a_{1} a_{2} \cdots a_{\bar{N}}\right\} ; \forall k, l \in\{1,2, \ldots, t\}, b_{k} \neq b_{l}$ as $\mho$, where $(\cdot)$ ! is the factorial of variables and $b_{1} b_{2} \cdots b_{t}$ can be expressed as follows:

$$
\begin{gathered}
b_{1}=e^{-m_{1} y / \bar{\gamma}_{1}} \cdot \sum_{j=0}^{m_{1}-1} j !\left(\frac{m_{1} y}{\bar{\gamma}_{1}}\right)^{j} \\
b_{2}=e^{-m_{2} y / \bar{\gamma}_{2}} \cdot \sum_{j=0}^{m_{2}-1} j !\left(\frac{m_{2} y}{\bar{\gamma}_{2}}\right)^{j} \\
\vdots \\
b_{t}=e^{-m_{t} y / \bar{\gamma}_{t}} \cdot \sum_{j=0}^{m_{t}-1} j !\left(\frac{m_{t} y}{\bar{\gamma}_{t}}\right)^{j} .
\end{gathered}
$$

By substituting (10) and (11) into (9), we have the expression of the PDF for the received SNR via the multihop relaying link as
With (12) as the PDF of the received SNR at the destination from the multihop caching link as well as the Nakagami distribution of the direct wireless channel from the source to the destination, the total SNR received at the destination node after the MRC receiver can be expressed as

$$
\begin{gathered}
f_{z}(z)=\int_{0}^{z} f_{X}(x) f_{Y}(z-x) d x=\frac{1}{\Gamma(\lambda)}\left(\frac{\lambda}{\Omega}\right)^{\lambda} \\
\cdot \sum_{s=0}^{\lambda-1} C_{\lambda-1}^{s} z^{\lambda-1-s}(-1)^{s} e^{-(\lambda / \Omega) z} \int_{0}^{z} f_{X}(x) \cdot e^{(\lambda / \Omega) x} \\
\cdot x^{s} d x=\frac{\lambda^{\lambda} \cdot e^{-(\lambda / \Omega) z} \cdot C_{\lambda-1}^{s}}{(-1)^{s+t+1} \Gamma(\lambda) \Omega^{\lambda} z^{1+s-\lambda}} \sum_{s=0}^{\lambda-1} \sum_{t=0}^{N} \sum_{\mho} \sum_{j_{1}=0}^{m_{1}-1} \sum_{j_{2}=0}^{m_{2}-1} \\
\quad \ldots \sum_{j_{t}=0}^{m_{t}-1} \frac{\left(m_{1} / \bar{\gamma}_{1}\right)^{j_{1}} \cdots\left(m_{t} / \bar{\gamma}_{t}\right)^{j_{t}}}{j_{1} ! \cdots j_{t} !} \cdot\left\{\int_{0}^{z} \sum_{q=1}^{t} j_{q}\right.
\end{gathered}
$$




$$
\begin{aligned}
& \cdot\left(\sum_{p=1}^{t} \frac{m_{p}}{\bar{\gamma}_{p}}-\frac{\lambda}{\Omega}\right)^{-\left(s+1+\sum_{q=1}^{t} j_{q}\right)} \\
& \left.\cdot \gamma\left(s+1+\sum_{q=1}^{t} j_{q},\left(\sum_{p=1}^{t} \frac{m_{p}}{\bar{\gamma}_{p}}-\frac{\lambda}{\Omega}\right) \cdot z\right)\right\} .
\end{aligned}
$$

The parameter lambda is the shadowing effect value and can be expressed as $\lambda=1 /\left(e^{\left(\sigma_{d B} / 8.686\right)^{2}}-1\right)$, where $\sigma_{d B}$ is the shadow spread parameter expressed in decibels whose value usually ranges from 4 to 9 in practice. The $\Gamma(\lambda)$ can be expressed as $\Gamma(\lambda)=\int_{0}^{\infty} t^{\lambda-1} e^{-t} d t$, where symbol $C$ is for "Combination." A combination is an unordered collection of distinct elements and usually of a prescribed size and taken from a given set such as $C_{n}^{r}=n ! / r !(n-r)$ ! (! denotes factorial). It is observed that there are two terms involving the incomplete gamma function and the expression can be derived using some manipulations [28] as

$$
\begin{aligned}
& \gamma\left(\sum_{q=1}^{t} j_{q}+s,\left(\sum_{p=1}^{t} \frac{m_{p}}{\bar{\gamma}_{p}}-\frac{\lambda}{\Omega}\right) \cdot z\right) \\
&= \Gamma\left(\sum_{q=1}^{t} j_{q}+s\right) \\
& \cdot\left[1-\sum_{k=0}^{\sum_{q=1}^{t} j_{q}+s} \frac{\left(\sum_{p=1}^{t}\left(m_{p} / \bar{\gamma}_{p}\right)-\lambda / \Omega\right)^{k} \cdot z^{k}}{e^{\left(\sum_{p=1}^{t}\left(m_{p} / \bar{\gamma}_{p}\right)-\lambda / \Omega\right) \cdot z} k !}\right] . \\
& \gamma\left(s+1+\sum_{q=1}^{t} j_{q},\left(\sum_{p=1}^{t} \frac{m_{p}}{\bar{\gamma}_{p}}-\frac{\lambda}{\Omega}\right) \cdot z\right) \\
&=\Gamma\left(s+1+\sum_{q=1}^{t} j_{q}\right) \\
& \cdot\left[1-\sum_{l=0}^{s+1+\sum_{q=1}^{t} j_{q}} \frac{\left(\sum_{p=1}^{t}\left(m_{p} / \bar{\gamma}_{p}\right)-\lambda / \Omega\right)^{l} \cdot z^{l}}{e^{\left(\sum_{p=1}^{t}\left(m_{p} / \bar{\gamma}_{p}\right)-\lambda / \Omega\right) \cdot z} \cdot l !}\right]
\end{aligned}
$$

By substituting (14) into (13), we can obtain the outage probability expression of the received SNR at the destination node after the MRC receiver as

$$
\begin{gathered}
P_{\text {out }}\left(\eta_{\text {th }}\right)=\frac{\lambda^{\lambda} C_{\lambda-1}^{s} z^{\lambda-1-s}}{(-1)^{s+t+1} \Gamma(\lambda) \Omega^{\lambda}} \sum_{s=0}^{\lambda-1} \sum_{t=0}^{\bar{N}} \sum_{\mho} \sum_{j_{1}=0}^{m_{1}-1} \sum_{j_{2}=0}^{m_{2}-1} \\
\ldots \sum_{j_{t}=0}^{m_{t}-1} \frac{\left(m_{1} / \bar{\gamma}_{1}\right)^{j_{1}} \cdots\left(m_{t} / \bar{\gamma}_{t}\right)^{j_{t}}}{j_{1} ! \cdots j_{t} !} \cdot\left\{\sum _ { q = 1 } ^ { t } j _ { q } \cdot \left(\sum_{p=1}^{t} \frac{m_{p}}{\bar{\gamma}_{p}}\right.\right. \\
\left.-\frac{\lambda}{\Omega}\right)^{-\left(\sum_{q=1}^{t} j_{q}+s\right)} \cdot \Gamma\left(\sum_{q=1}^{t} j_{q}+s\right) \cdot \int_{0}^{\eta_{\mathrm{th}}}\left[e^{-(\lambda / \Omega) z}\right.
\end{gathered}
$$

$$
\begin{aligned}
& -e^{-\left(\sum_{p=1}^{t}\left(m_{p} / \bar{\gamma}_{p}\right)\right) \cdot z} \\
& \left.\cdot \sum_{k=0}^{\sum_{q=1}^{t} j_{q}+s} \frac{\left(\sum_{p=1}^{t}\left(m_{p} / \bar{\gamma}_{p}\right)-\lambda / \Omega\right)^{k} \cdot z^{k}}{k !}\right]-\sum_{p=1}^{t} \frac{m_{p}}{\bar{\gamma}_{p}} \\
& \cdot\left(\sum_{p=1}^{t} \frac{m_{p}}{\bar{\gamma}_{p}}-\frac{\lambda}{\Omega}\right)^{-\left(s+1+\sum_{q=1}^{t} j_{q}\right)} \cdot \Gamma\left(s+1+\sum_{q=1}^{t} j_{q}\right) \\
& \cdot \int_{0}^{\eta_{\text {th }}}\left[e^{-(\lambda / \Omega) z}-e^{-\left(\sum_{p=1}^{t}\left(m_{p} / \bar{\gamma}_{p}\right)\right) \cdot z}\right. \\
& \left.\left.\cdot \sum_{l=0}^{s+1+\sum_{q=1}^{t} j_{q}} \frac{\left(\sum_{p=1}^{t}\left(m_{p} / \bar{\gamma}_{p}\right)-\lambda / \Omega\right)^{l} \cdot z^{l}}{l !}\right]\right\} \\
& =\frac{\lambda^{\lambda} C_{\lambda-1}^{s} z^{\lambda-1-s}}{(-1)^{s+t+1} \Gamma(\lambda) \Omega^{\lambda}} \sum_{s=0}^{\lambda-1} \sum_{t=0}^{N} \sum_{\mho} \sum_{j_{1}=0}^{m_{1}-1} \sum_{j_{2}=0}^{m_{2}-1} \\
& \cdots \sum_{j_{t}=0}^{m_{t}-1} \frac{\left(m_{1} / \bar{\gamma}_{1}\right)^{j_{1}} \cdots\left(m_{t} / \bar{\gamma}_{t}\right)^{j_{t}}}{j_{1} ! \cdots j_{t} !} \cdot\left\{\sum_{q=1}^{t} j_{q}\right. \\
& \cdot \frac{\Gamma\left(\sum_{q=1}^{t} j_{q}+s\right)}{\left(\sum_{p=1}^{t}\left(m_{p} / \bar{\gamma}_{p}\right)-\lambda / \Omega\right)^{\left(\sum_{q=1}^{t} j_{q}+s\right)}} \cdot\left[\frac{\Omega}{\lambda}(1\right. \\
& \left.-e^{-(\lambda / \Omega) \eta_{\mathrm{th}}}\right)-\sum_{k=0}^{s+\sum_{q=1}^{t} j_{q}} \frac{\left(\sum_{p=1}^{t}\left(m_{p} / \bar{\gamma}_{p}\right)-\lambda / \Omega\right)^{k}}{k !\left(\sum_{p=1}^{t}\left(m_{p} / \bar{\gamma}_{p}\right)\right)^{k+1}} \\
& \left.\cdot \gamma\left(k+1, \sum_{p=1}^{t} \frac{m_{p}}{\bar{\gamma}_{p}} \cdot \eta_{\mathrm{th}}\right)\right]-\sum_{p=1}^{t} \frac{m_{p}}{\bar{\gamma}_{p}} \\
& \cdot \frac{\Gamma\left(s+1+\sum_{q=1}^{t} j_{q}\right)}{\left(\sum_{p=1}^{t}\left(m_{p} / \bar{\gamma}_{p}\right)-\lambda / \Omega\right)^{\left(s+1+\sum_{q=1}^{t} j_{q}\right)}} \cdot\left[\frac{\Omega}{\lambda}(1\right. \\
& \left.-e^{-(\lambda / \Omega) \eta_{\mathrm{th}}}\right)-\sum_{l=0}^{s+1+\sum_{q=1}^{t} j_{q}} \frac{\left(\sum_{p=1}^{t}\left(m_{p} / \bar{\gamma}_{p}\right)-\lambda / \Omega\right)^{l}}{l !\left(\sum_{p=1}^{t}\left(m_{p} / \bar{\gamma}_{p}\right)\right)^{l+1}} \\
& \left.\left.\cdot \gamma\left(k+1, \sum_{p=1}^{t} \frac{m_{p}}{\bar{\gamma}_{p}} \cdot \eta_{\mathrm{th}}\right)\right]\right\} .
\end{aligned}
$$

Based on (15), the bit error rate of the whole system can be expressed as

$$
P_{\mathrm{BER}}=E\{Q(\sqrt{v \gamma})\}=\frac{\sqrt{v}}{2 \sqrt{2 \pi}} \int_{0}^{\infty} \frac{e^{-(v / 2) x}}{\sqrt{x}} F(x) d x,
$$


where the function $Q(\cdot)$ is the Gaussian tail function and $v$ is the modulation mode in the transmission process; for example, $v=1$ represents the BFSK modulation and $v=2$ is the BPSK modulation. have

By substituting the CDF of received SNR into (16), we can

$$
\begin{aligned}
& P_{\mathrm{BER}}=\frac{\sqrt{v}}{2 \sqrt{2 \pi}} \int_{0}^{\infty} \frac{e^{-(v / 2) x}}{\sqrt{x}} \frac{\lambda^{\lambda} C_{\lambda-1}^{s} z^{\lambda-1-s}}{(-1)^{s+t+1} \Gamma(\lambda) \Omega^{\lambda}} \\
& \cdot \sum_{s=0}^{\lambda-1} \sum_{t=0}^{\bar{N}} \sum_{\mho} \sum_{j_{1}=0}^{m_{1}-1} \sum_{j_{2}=0}^{m_{2}-1} \cdots \sum_{j_{t}=0}^{m_{t}-1} \frac{\left(m_{1} / \bar{\gamma}_{1}\right)^{j_{1}} \cdots\left(m_{t} / \bar{\gamma}_{t}\right)^{j_{t}}}{j_{1} ! \cdots j_{t} !} \\
& \left\{\sum_{q=1}^{t} j_{q} \cdot \frac{\Gamma\left(\sum_{q=1}^{t} j_{q}+s\right)}{\left(\sum_{p=1}^{t}\left(m_{p} / \bar{\gamma}_{p}\right)-\lambda / \Omega\right)^{\left(\sum_{q=1}^{t} j_{q}+s\right)}}\right. \\
& \cdot\left[\frac{\Omega}{\lambda}\left(1-e^{-(\lambda / \Omega) \eta_{\mathrm{th}}}\right)\right. \\
& -\sum_{k=0}^{s+\sum_{q=1}^{t} j_{q}} \frac{\left(\sum_{p=1}^{t}\left(m_{p} / \bar{\gamma}_{p}\right)-\lambda / \Omega\right)^{k}}{k !\left(\sum_{p=1}^{t}\left(m_{p} / \bar{\gamma}_{p}\right)\right)^{k+1}} \cdot \gamma(k \\
& \left.\left.+1, \sum_{p=1}^{t} \frac{m_{p}}{\bar{\gamma}_{p}} \cdot \eta_{\mathrm{th}}\right)\right]-\sum_{p=1}^{t} \frac{m_{p}}{\bar{\gamma}_{p}} \\
& \cdot \frac{\Gamma\left(s+1+\sum_{q=1}^{t} j_{q}\right)}{\left(\sum_{p=1}^{t}\left(m_{p} / \bar{\gamma}_{p}\right)-\lambda / \Omega\right)^{\left(s+1+\sum_{q=1}^{t} j_{q}\right)}} \cdot\left[\frac{\Omega}{\lambda}(1\right. \\
& \left.-e^{-(\lambda / \Omega) \eta_{\mathrm{th}}}\right)-\sum_{l=0}^{s+1+\sum_{q=1}^{t} j_{q}} \frac{\left(\sum_{p=1}^{t}\left(m_{p} / \bar{\gamma}_{p}\right)-\lambda / \Omega\right)^{l}}{l !\left(\sum_{p=1}^{t}\left(m_{p} / \bar{\gamma}_{p}\right)\right)^{l+1}} \\
& \left.\left.\cdot \gamma\left(k+1, \sum_{p=1}^{t} \frac{m_{p}}{\bar{\gamma}_{p}} \cdot \eta_{\mathrm{th}}\right)\right]\right\} d x \\
& \wp=\frac{\Omega}{\lambda} \cdot\left(\sqrt{\frac{2 \pi}{v}}-\sqrt{\frac{\pi}{v / 2+\lambda / \Omega}}\right)
\end{aligned}
$$$$
\cdot \frac{\sqrt{v} \lambda^{\lambda} C_{\lambda-1}^{s} z^{\lambda-1-s}(-1)^{s+t+1}}{2 \sqrt{2 \pi} \Gamma(\lambda) \Omega^{\lambda}} \sum_{s=0}^{\lambda-1} \sum_{t=0}^{N} \sum_{\mho} \sum_{j_{1}=0}^{m_{1}-1} \sum_{j_{2}=0}^{m_{2}-1}
$$$$
\cdots \sum_{j_{t}=0}^{m_{t}-1} \frac{\left(m_{1} / \bar{\gamma}_{1}\right)^{j_{1}} \cdots\left(m_{t} / \bar{\gamma}_{t}\right)^{j_{t}}}{j_{1} ! \cdots j_{t} !}
$$$$
\left\{\frac{\Gamma\left(\sum_{q=1}^{t} j_{q}+s\right) \cdot \sum_{q=1}^{t} j_{q}}{\left(\sum_{p=1}^{t}\left(m_{p} / \bar{\gamma}_{p}\right)-\lambda / \Omega\right)^{\left(\sum_{q=1}^{t} j_{q}+s\right)}} \cdot[\wp\right.
$$

$$
\begin{aligned}
& -\sum_{k=0}^{s+\sum_{q=1}^{t} j_{q}} \frac{\left(\sum_{p=1}^{t}\left(m_{p} / \bar{\gamma}_{p}\right)-\lambda / \Omega\right)^{k}}{k !\left(\sum_{p=1}^{t}\left(m_{p} / \bar{\gamma}_{p}\right)\right)^{k+1}} \\
& {\left[\sqrt{\frac{2 \pi}{v}} \Gamma(k+1)\right.} \\
& \left.\left.-\sum_{r_{1}=0}^{k} \frac{\left(\sum_{p=1}^{t}\left(m_{p} / \bar{\gamma}_{p}\right)\right)^{r_{1}} \cdot \Gamma\left(r_{1}+1 / 2\right)}{\left(v / 2+\sum_{p=1}^{t}\left(m_{p} / \bar{\gamma}_{p}\right)\right)^{r_{1}+1 / 2}}\right]\right] \\
& -\frac{\Gamma\left(s+1+\sum_{q=1}^{t} j_{q}\right) \cdot \sum_{p=1}^{t}\left(m_{p} / \bar{\gamma}_{p}\right)}{\left(\sum_{p=1}^{t}\left(m_{p} / \bar{\gamma}_{p}\right)-\lambda / \Omega\right)^{\left(s+1+\sum_{q=1}^{t} j_{q}\right)}} \cdot[\wp \\
& -\sum_{l=0}^{s+1+\sum_{q=1}^{t} j_{q}} \frac{\left(\sum_{p=1}^{t}\left(m_{p} / \bar{\gamma}_{p}\right)-\lambda / \Omega\right)^{l}}{l !\left(\sum_{p=1}^{t}\left(m_{p} / \bar{\gamma}_{p}\right)\right)^{l+1}} \\
& {\left[\sqrt{\frac{2 \pi}{v}} \Gamma(l+1)\right.} \\
& \left.\left.-\sum_{r_{2}=0}^{l} \frac{\left(\sum_{p=1}^{t}\left(m_{p} / \bar{\gamma}_{p}\right)\right)^{r_{2}} \cdot \Gamma\left(r_{2}+1 / 2\right)}{\left(v / 2+\sum_{p=1}^{t}\left(m_{p} / \bar{\gamma}_{p}\right)\right)^{r_{2}+1 / 2}}\right]\right] .
\end{aligned}
$$

To this end, the outage probability and BER closed form analytical expression are derived in detail, which are suitable for the Nakagami channel in the transmission process. Moreover, the bit error rate is expressed in the closed form without any approximation, which is different from the existing results in this literature.

\section{Simulation and Analysis}

In this section, we present the various performance evaluation results derived by numerical and simulations with a binary phase shift keying modulation scheme. We also verify the gap between the derived theoretical results and the numerical simulations. We assume that the transmitting power of source terminal and relay terminal are the same in general. We consider that the multihop network is composed by a LoS path and three relays. The relays employ the amplifyand-forward technique and each node is equipped with only one antenna. The simulations operate over Nakagami-m fading channel with different fading parameter $m$. We employ the maximal-ratio combining at the destination.

Figure 2 plots the outage probability function versus the SNR received at the destination, where different channel distributions of the direct link from the source to the destination are simulated for the different Nakagami factor, respectively. It is shown that the derived theoretical result is very tight to the curve from the numerical simulations in the scenario of $m=1,2,3$ for every SNR. Thus, it is concluded that our result is very accurate from the outage probability 


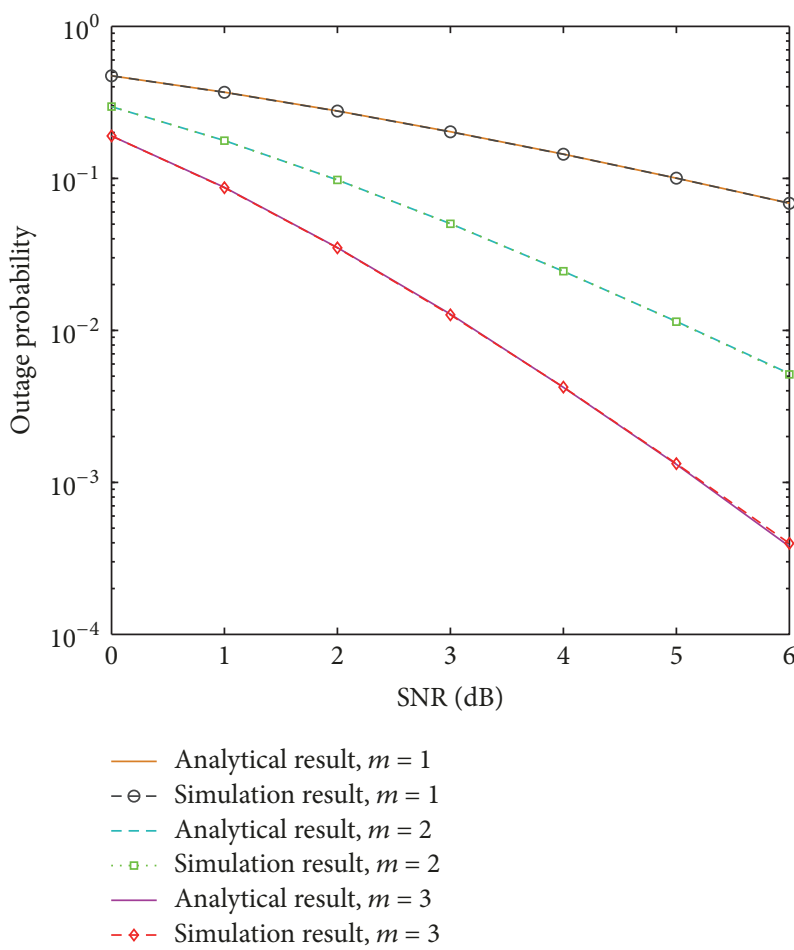

FIGURE 2: The outage probability.

point of view. It is mentioned that the outage probability can be decreased with the $m$ growing, because the parameter $m$ stands for the strength of line of sight, so the bigger the factor $m$, the better the system performance.

Figure 3 gives the bit error rate as the function of the SNR received at the destination. It is seen from Figure 3 that our derived theoretical expression is almost the same with the result from the numerical simulations in the every regime of SNR regardless of the Nakagami factor $m$. Thus, it is concluded that our scheme is very accurate from the BER point of view. It is worth noting that the BER of multihop network can be decreased with the relay increasing; the reason is that the relay can amplify the received signal and forward to the next node and the multiple relay can reduce the large scale fading to some extent. However, the broadcast error with relay node is not considered herein and will be our future work.

Figure 4 plots the outage probability comparison of LoS and non-LoS. It can be seen that the performance with LoS is superior to the non-LoS; the reason is that LoS can improve the received SNR and increase the diversity order for the IoT networks. Figure 5 presents the outage probability comparison with different parameter; we can see that the bigger the parameter $m$, the better the outage probability performance. The reason is that the parameter $m$ represents the strength of LoS, which can result in the higher received SNR.

Figure 6 shows the BER comparison with different parameter; it seems that the less relay number presents more superior BER performance; the reason is that the multihop IoT network may bring about different cases for each link;

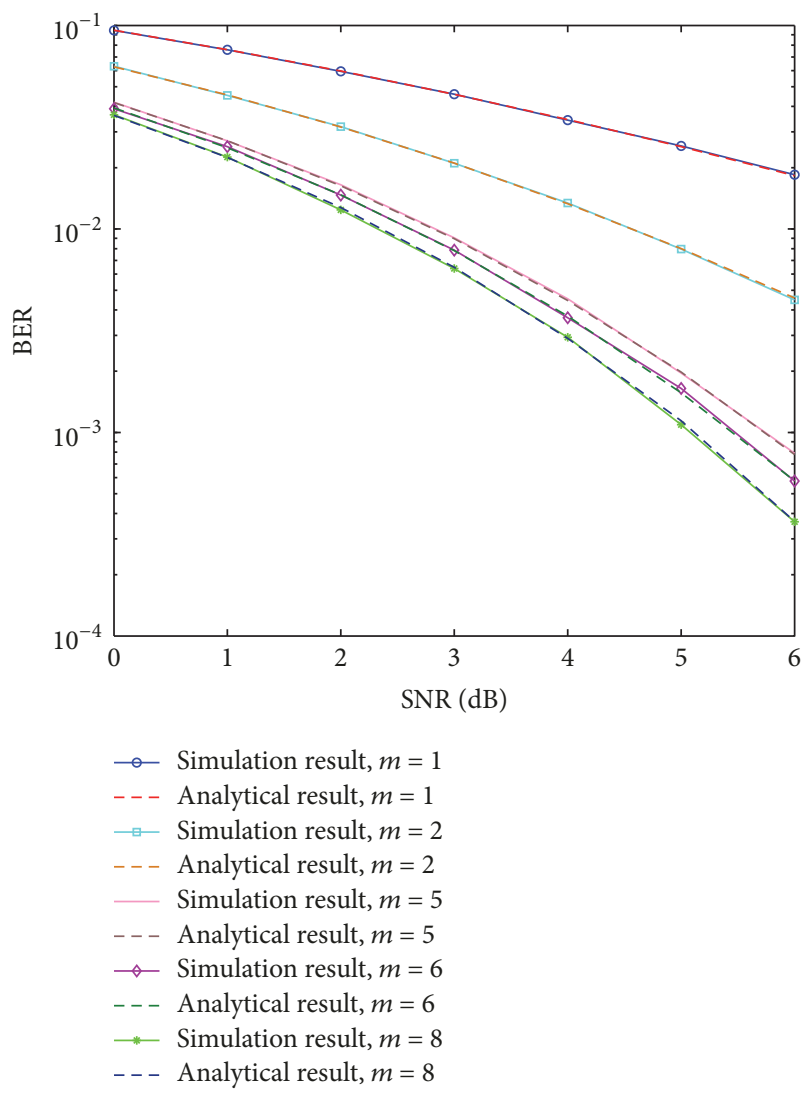

FIGURE 3: The BER performance of the IoT network.

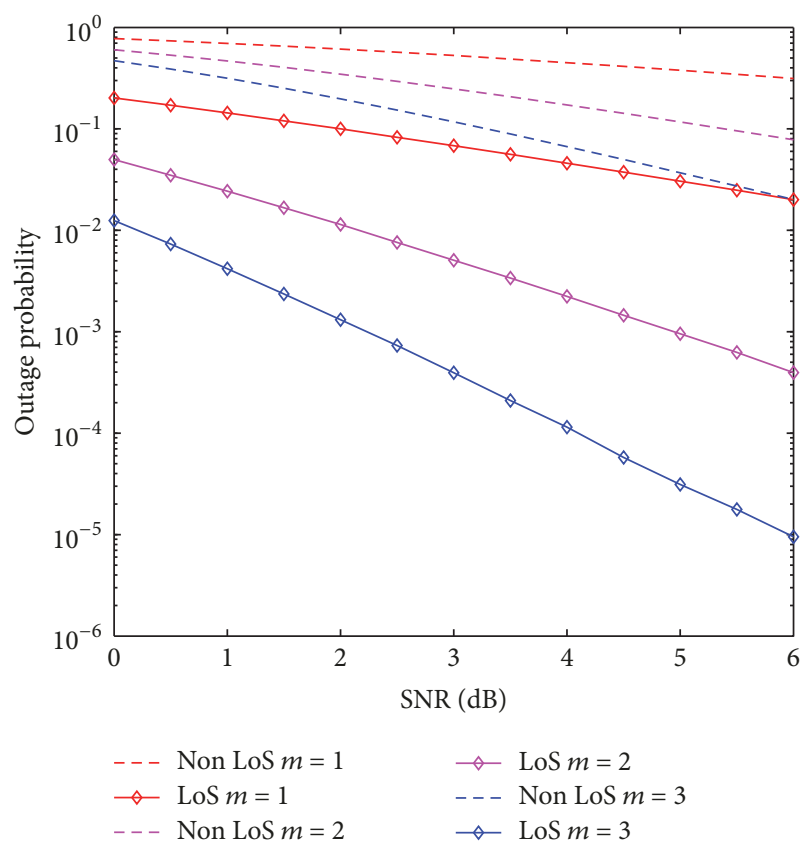

FIGURE 4: The outage probability comparison of LoS and non-LoS.

however, the IoT network may depend on the multihop relay nodes to improve the communication efficiency. 


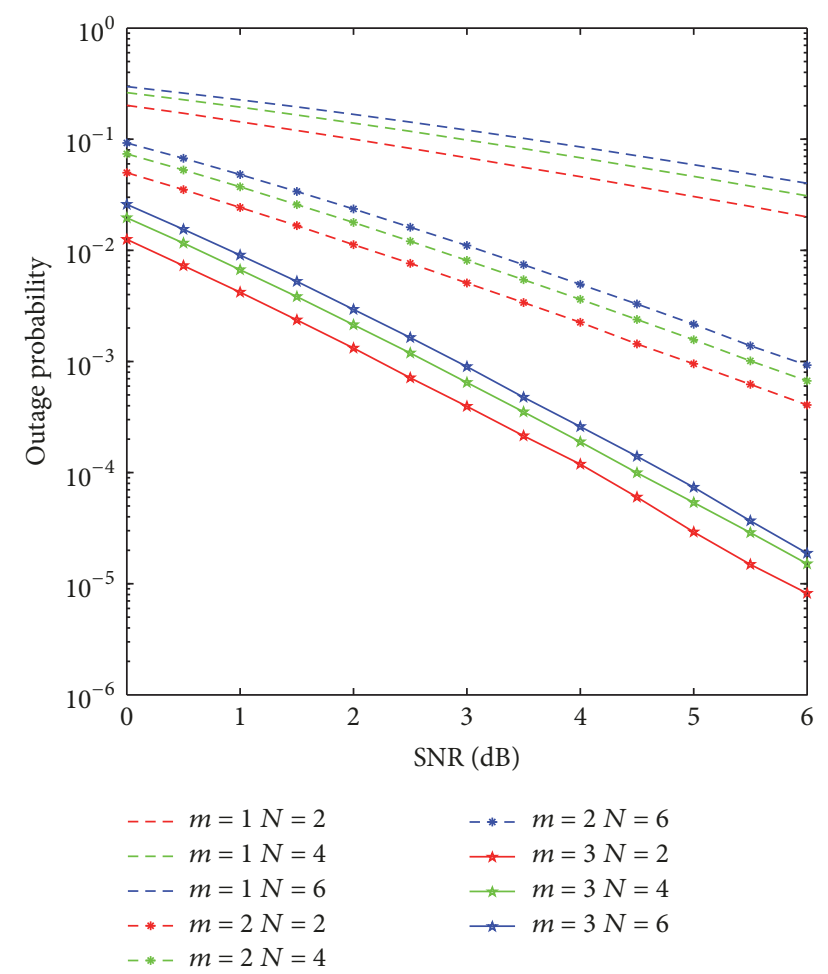

FIGURE 5: The outage probability comparison with different parameter.

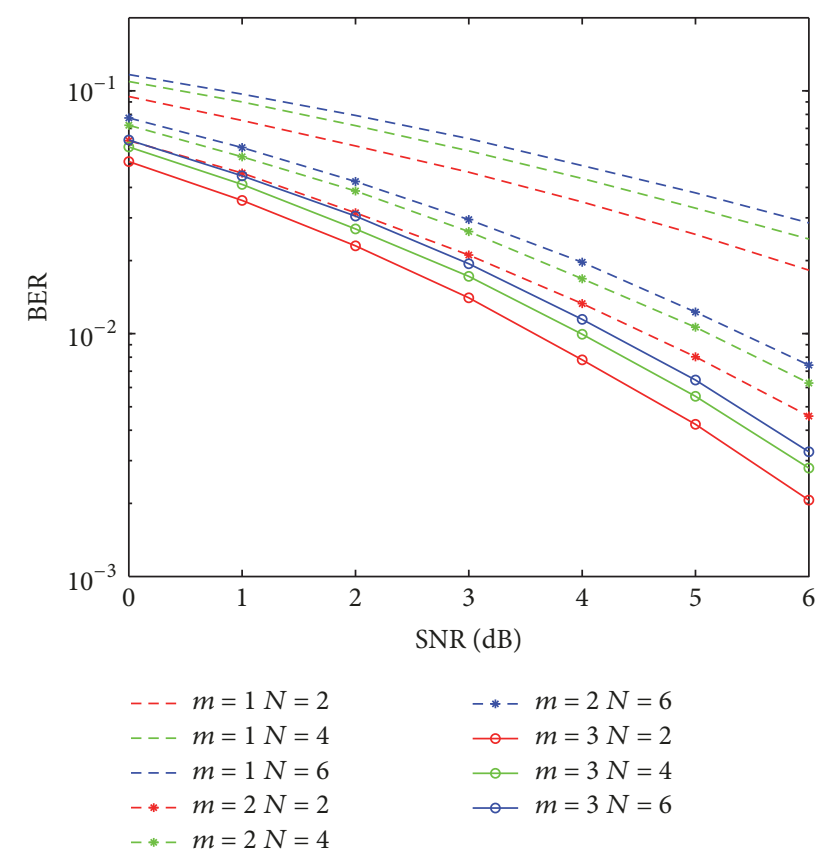

FIGURE 6: The BER comparison with different parameter.

\section{Conclusions}

In this paper, the probability distribution function is derived in closed form expression without any approximation for the signal-to-noise ratio received at the destination node from the multihop caching link in Internet of Things system.
Moreover, both the outage probability function and the bit error rate are derived in analytical expressions by exploiting the function and integration properties. The obtained results are suitable for any distribution of the wireless channel from the source directly to the destination node in IoT communications.

\section{Conflicts of Interest}

The authors declare that they have no conflicts of interest.

\section{Acknowledgments}

This work was supported by the National Natural Science Foundation of China under Grants U1404615, 61671144, 41605122, 61701172, and U1504619; National Thirteen Five National Defense Fund under Grant 6140311030207; Project of Education Department Cooperation Cultivation under Grant 201602011005; China Postdoctoral Science Foundation under Grant 2015M571637; Open Funds of Key Laboratory of Middle Atmosphere and Global Environment Observation; Open Funds of State Key Laboratory of Millimeter Waves under Grant K201504; Program for Science \& Technology Innovation Talents in the University of Henan Province (Educational Committee) of China (17HASTIT025); Program for International Science \& Technology Cooperation Projects of Henan Province (172102410072); Shandong Province Natural Science Foundation (ZR2017BF028).

\section{References}

[1] N. Zhao, S. Zhang, F. R. Yu, Y. Chen, A. Nallanathan, and V. C. M. Leung, "Exploiting Interference for Energy Harvesting: A Survey, Research Issues, and Challenges," IEEE Access, vol. 5, pp. 10403-10421, 2017.

[2] N. Zhao, F. Richard Yu, M. Jin, Q. Yan, and V. C. M. Leung, "Interference Alignment and Its Applications: A Survey, Research Issues, and Challenges," IEEE Communications Surveys \& Tutorials, vol. 18, no. 3, pp. 1779-1803, 2016.

[3] Q. Wu, G. Y. Li, W. Chen, D. W. K. Ng, and R. Schober, "An Overview of Sustainable Green 5G Networks," IEEE Wireless Communications Magazine, vol. 24, no. 4, pp. 72-80, 2017.

[4] S. Zhang, Q. Wu, S. Xu, and G. Y. Li, "Fundamental Green Tradeoffs: Progresses, Challenges, and Impacts on 5G Networks," IEEE Communications Surveys \& Tutorials, vol. 19, no. 1, pp. 33-56, 2017.

[5] P. Solic, Z. Blazevic, M. Skiljo, L. Patrono, R. Colella, and J. J. P. C. Rodrigues, "Gen2 RFID as IoT enabler: Characterization and performance improvement," IEEE Wireless Communications Magazine, vol. 24, no. 3, article no. 2402, pp. 33-39, 2017.

[6] W. Li, C. Zhu, V. C. M. Leung, L. T. Yang, and Y. Ma, "Performance comparison of cognitive radio sensor networks for industrial IoT with different deployment patterns," IEEE Systems Journal, vol. 11, no. 3, pp. 1456-1466, 2015.

[7] N. Kaur and S. K. Sood, "A game theoretic approach for an IoT-based automated employee performance evaluation," IEEE Systems Journal, vol. 11, no. 3, pp. 1385-1394, 2017.

[8] D. Chen, J. Cong, S. Gurumani, W. Hwu, K. Rupnow, and Z. Zhang, "Platform choices and design demands for IoT 
platforms: cost, power, and performance tradeoffs," IET CyberPhysical Systems: Theory \& Applications, vol. 1, no. 1, pp. 70-77, 2016.

[9] Y. Zhang, W. Wang, N. Wu, and C. Qian, "IoT-Enabled RealTime Production Performance Analysis and Exception Diagnosis Model," IEEE Transactions on Automation Science and Engineering, vol. 13, no. 3, pp. 1318-1332, 2016.

[10] Q. Wu, M. Tao, D. W. Kwan Ng, W. Chen, and R. Schober, "Energy-Efficient Resource Allocation for Wireless Powered Communication Networks," IEEE Transactions on Wireless Communications, vol. 15, no. 3, pp. 2312-2327, 2016.

[11] D. Zucchetto and A. Zanella, "Uncoordinated Access Schemes for the IoT: Approaches, Regulations, and Performance," IEEE Communications Magazine, vol. 55, no. 9, pp. 48-54, 2017.

[12] T. Song, R. Li, B. Mei, J. Yu, X. Xing, and X. Cheng, "A Privacy Preserving Communication Protocol for IoT Applications in Smart Homes," IEEE Internet of Things Journal, vol. 4, no. 6, pp. 1844-1852, 2017.

[13] J. Shen, A. Wang, C. Wang, P. C. K. Hung, and C.-F. Lai, "An Efficient Centroid-Based Routing Protocol for Energy Management in WSN-Assisted IoT,' IEEE Access, vol. 5, pp. 1846918479, 2017

[14] M. Ashraf, A. Shahid, J. W. Jang, and K.-G. Lee, "Energy Harvesting Non-Orthogonal Multiple Access System with MultiAntenna Relay and Base Station," IEEE Access, vol. 5, pp. 1766017670, 2017

[15] H. Y. Lateef, M. Ghogho, and D. McLernon, "On the performance analysis of multi-hop cooperative relay networks over generalized-K fading channels," IEEE Communications Letters, vol. 15, no. 9, pp. 968-970, 2011.

[16] N. C. Beaulieu and S. S. Soliman, "Exact analytical solution for end-to-end SNR of multihop AF relaying systems," in Proceedings of the 2011 IEEE GLOBECOM Workshops, GC Wkshps 2011, pp. 580-585, USA, December 2011.

[17] H. A. Suraweera, P. J. Smith, and J. Armstrong, "Outage probability of cooperative relay networks in Nakagami-m fading channels," IEEE Communications Letters, vol. 10, no. 12, pp. 834836, 2006.

[18] G. Farhadi and N. C. Beaulieu, "Ergodic capacity analysis of wireless relaying systems in Rayleigh fading," in Proceedings of the IEEE International Conference on Communications, ICC 2008, pp. 3730-3735, chn, May 2008.

[19] G. Farhadi and N. C. Beaulieu, "On the outage and error probability of amplify-and-forward multi-hop diversity transmission systems," in Proceedings of the IEEE International Conference on Communications, ICC 2008, pp. 3748-3754, China, May 2008.

[20] K. Song, B. Ji, Y. Huang, M. Xiao, and L. Yang, "Performance analysis of antenna selection in two-way relay networks," IEEE Transactions on Signal Processing, vol. 63, no. 10, pp. 2520-2532, 2015.

[21] S. N. Datta and S. Chakrabarti, "Unified error analysis of dualhop relay link in Nakagami-m fading channels," IEEE Communications Letters, vol. 14, no. 10, pp. 897-899, 2010.

[22] B. Ji, K. Song, Y. Huang, and L. Yang, "A cooperative relay selection for two-way cooperative relay networks in nakagami channels," Wireless Personal Communications, vol. 71, no. 3, pp. 20452065, 2013.

[23] N. Zhao, X. Zhang, F. R. Yu, and V. C. M. Leung, “To Align or Not to Align: Topology Management in Asymmetric Interference Networks," IEEE Transactions on Vehicular Technology, vol. 66, no. 8, pp. 7164-7177, 2017.
[24] X. Ge, K. Huang, C. X. Wang, X. Hong, and X. Yang, "Capacity analysis of a multi-cell multi-antenna cooperative cellular network with co-channel interference," IEEE Transactions on Wireless Communications, vol. 10, no. 10, pp. 3298-3309, 2011.

[25] B. Ji, K. Song, C. Li, W. Zhu, and L. Yang, "Energy harvest and information transmission design in internet-of-things wireless communication systems," AEU - International Journal of Electronics and Communications, vol. 87, pp. 124-127, 2018.

[26] Statistical Methods in Radio Wave Propagation, Elsevier, 1960.

[27] M. O. Hasna and M.-S. Alouini, "End-to-end performance of transmission systems with relays over Rayleigh-fading channels," IEEE Transactions on Wireless Communications, vol. 2, no. 6, pp. 1126-1131, 2003.

[28] I. S. Gradshteyn and I. M. Ryzhik, Table of Integrals, Series, and Products, Academic Press, Inc., San Diego, CA, USA, 7th edition, 1996. 


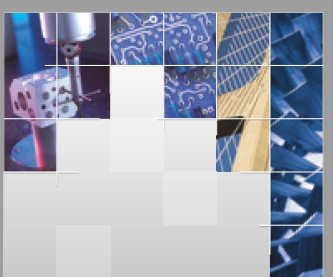

\section{Enfincering}
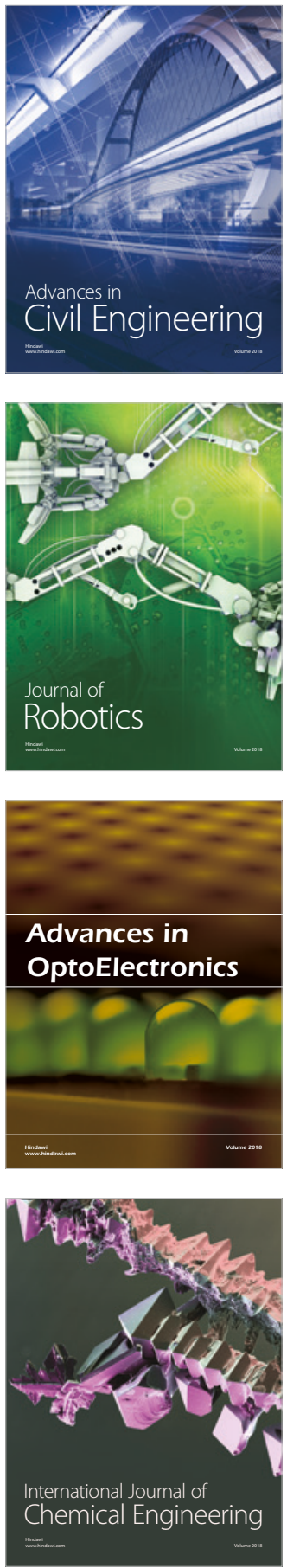

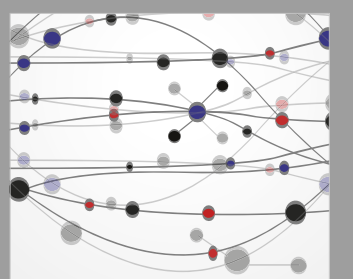

\section{Rotating \\ Machinery}

The Scientific World Journal

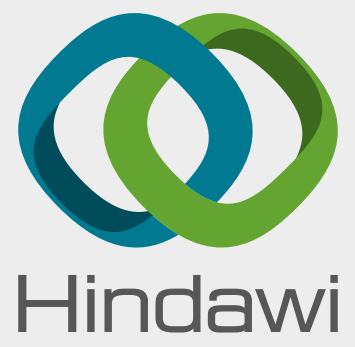

Submit your manuscripts at

www.hindawi.com
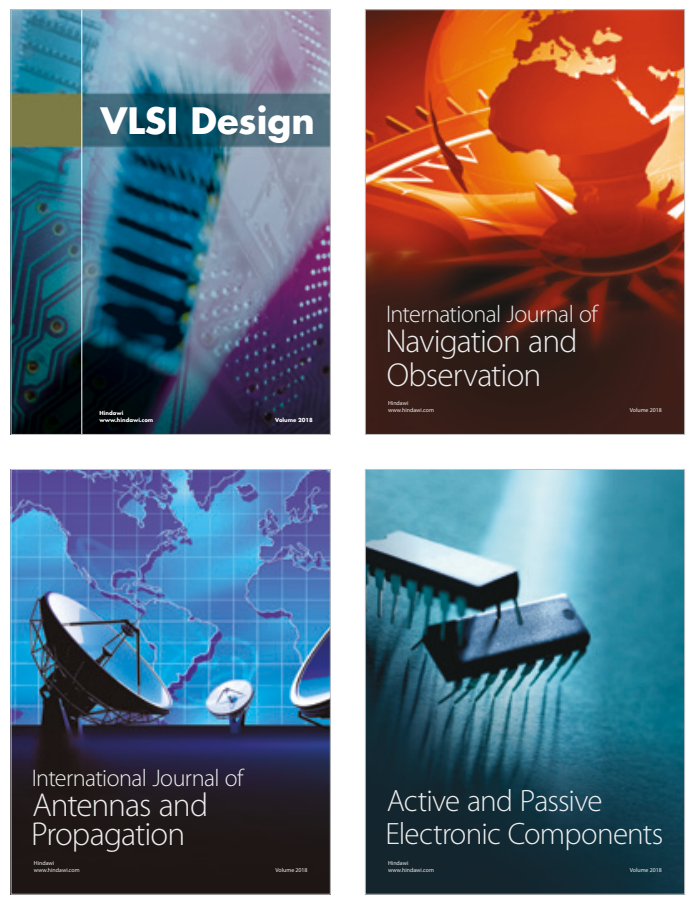
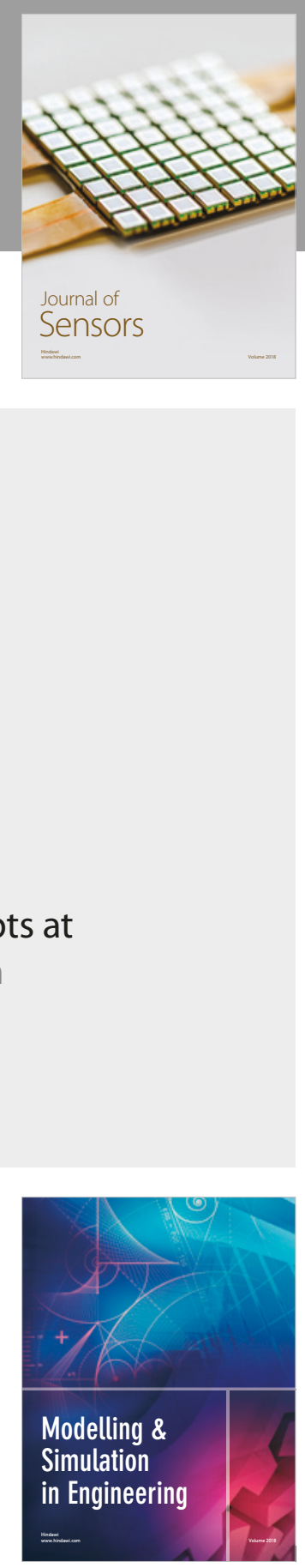

\section{Advances \\ Multimedia}
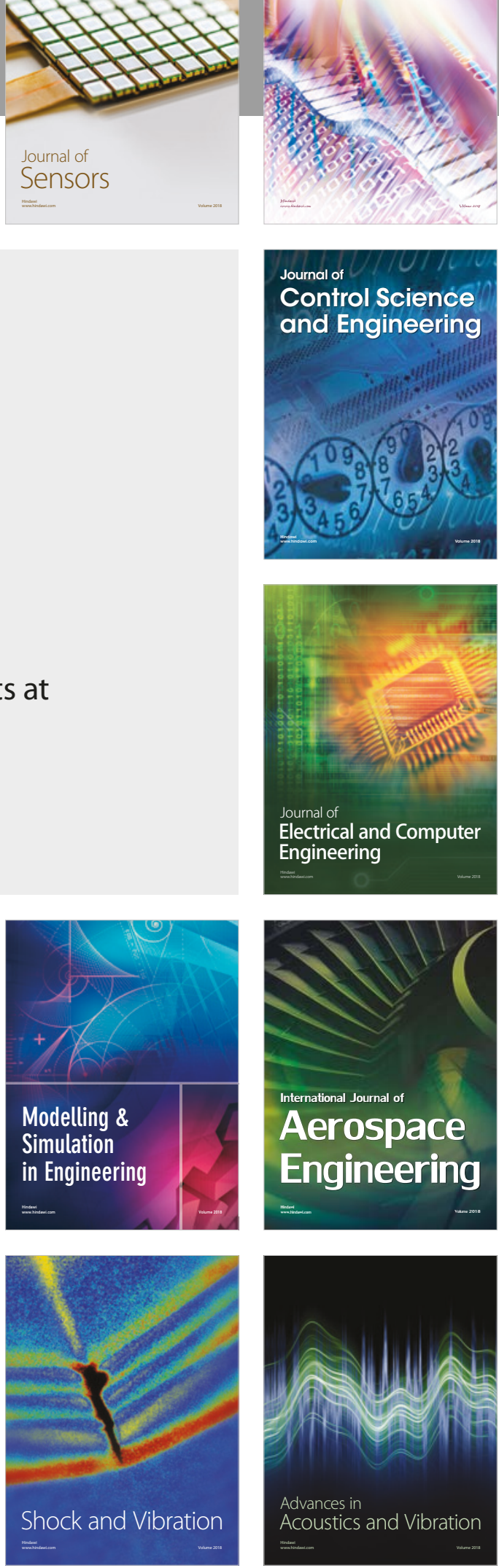